\title{
Division of Gynecologic Oncology
}

The Gynecologic Oncology Division at the University of lowa consists of four attending physicians, three fellows-intraining, two physician assistants, three nurses and supporting research and clerical staff. The concentration of our division remains the care of women with gynecologic malignancies. Our service area includes all counties of lowa as well as western Illinois. Our patients are seen and treated in the National Cancer Institute designated Holden Comprehensive Cancer Center on the University of lowa Hospitals campus.

The clinical work of our division includes surgery, chemotherapy and radiation therapy treatments. A current initiative of the division is minimally invasive surgery in women with uterine cancers. This has been lead by $\mathrm{Dr}$. David Bender who developed the robotic surgery program and trained Dr. Thomas Buekers in this technology. The division currently averages five procedures a week in women with gynecologic malignancies. A review of patient outcomes being assembled for publication has found significant decreases in patient length of stay and post operative complications with the use of robotic surgery.

The division continues its longstanding commitment to research through participation in clinical trials as well as a laboratory research initiative by Dr. Michael Goodheart. The division has been a member of the Gynecologic Oncology Group since shortly after its inception and continues to actively participate in trials. Our division was recently recognized as one of the five highest accruing sites among all GOG institutions. Dr. Goodheart is in the midst of a research training grant from the National Institutes of Health that allows him to divide his time between the clinic and laboratory. This year he was recognized for his work with a three-year, \$1.5 million grant award from the ARRA program.

Thomas E. Buekers, MD

Interim Director, Division of Gynecologic Oncology 